\title{
Enzymatic Functionalization of Cork Surface with Antimicrobial Hybrid Biopolymer/Silver Nanoparticles
}

Antonio Francesko ${ }^{\dagger, \#}$, Lucas Blandón ${ }^{\ddagger}, \#$, Mario Vázquez ${ }^{\star}$, Petya Petkova ${ }^{\dagger}$, Jordi Morató ${ }^{\S}$, Annett Pfeifer" ${ }^{\prime}$, Thomas Heinzel ${ }$, Ernest Mendoza ${ }^{\perp}$, Tzanko Tzanov ${ }^{\dagger, *}$

$\uparrow$ Grup de Biotecnologia Molecular i Industrial, Department of Chemical Engineering, Universitat Politècnica de Catalunya, Spain

† Grupo Interdisciplinario de Estudios Moleculares, Instituto de Química, Universidad de Antioquia, Medellín, Colombia

§ AQUASOST Group - UNESCO Chair on Sustainability, Universitat Politècnica de Catalunya, Spain

|| Center of Excellence for Polysaccharide Research, Institute of Organic Chemistry and Macromolecular Chemistry, Friedrich Schiller University of Jena, Germany

$\perp$ Grup de Nanomaterials Aplicats. Centre de Recerca en Nanoenginyeria, Universitat Politècnica de Catalunya, Spain

* Corresponding author: Dr. Tzanko Tzanov

Grup de Biotecnologia Molecular i Industrial, Department of Chemical Engineering, Universitat Politècnica de Catalunya, Rambla Sant Nebridi 22, 08222, Terrassa, Spain

Tel: +34937398570; Fax: +34937398225

E-mail: tzanko.tzanov@upc.edu

\# These authors contributed equally to the work 


\begin{abstract}
Laccase-assisted assembling of hybrid biopolymer-silver nanoparticles and cork matrices into an antimicrobial material with potential for water remediation is herein described. Amino-functional biopolymers were first used as doping agents to stabilize concentrated colloidal dispersions of silver nanoparticles (AgNPs), additionally providing the particles with functionalities for covalent immobilization onto cork to impart durable antibacterial effect. The solvent-free AgNPs synthesis by chemical reduction was carried out in presence of chitosan (CS) or 6-deoxy-6-( $\omega$ aminoethyl) aminocellulose (AC), leading to simultaneous AgNPs biofunctionalization. This approach resulted in concentrated hybrid NPs dispersion stable to aggregation and with hydrodynamic radius of particles of about $250 \mathrm{~nm}$. Moreover, laccase enabled coupling between the phenolic groups in cork and amino moieties in the biopolymer-doped AgNPs for permanent modification of the material. The antibacterial efficiency of the functionalized cork matrices, aimed as adsorbents for wastewater treatment, was evaluated against Escherichia coli and Staphylococcus aureus during 5 days in conditions mimicking those in constructed wetlands. Both intrinsically antimicrobial $\mathrm{CS}$ and $\mathrm{AC}$ contributed to the bactericidal effect of the enzymatically-grafted on cork AgNPs. In contrast, unmodified AgNPs were easily washed off from the material, confirming that the biopolymers potentiated a durable antibacterial functionalization of the cork matrices.
\end{abstract}

Keywords: amino-functional biopolymers, silver nanoparticles, cork, enzymatic grafting, antimicrobial activity 


\section{Introduction}

Inadequate waste treatment and poor infrastructure for water disinfection are threats to water quality and public health through disease outbreaks globally. Many decontamination strategies have been implemented with different success for reducing the impact of water pollution. Constructed wetlands in various designs are the most versatile systems for removal of pesticides, heavy metals, polycyclic aromatic compounds and pharmaceutical residues. ${ }^{1-4}$ Currently, a major issue with these systems is related to the additional microbial contamination, especially the growth of undesired pathogen bacteria. Reutilization of the water treated in wetlands, e.g. for irrigation, requires its previous microbial decontamination to meet the agricultural quality standards. In Spain, for example, a maximum of 100 UFC of Escherichia coli per $100 \mathrm{~mL}$ irrigation water are recommended. Nowadays, the development of efficient materials for microbiological remediation of wastewaters is being intensively investigated..$^{5-6}$ Silver nanoparticles (AgNPs) are largely claimed as broad-spectrum antibacterial agents, which efficacy depends on their size and morphology. While the mechanism of their antibacterial activity remains a subject of debate, the most widely accepted one involves the perturbation of the bacterial cell wall by the NPs, resulting in cell death. ${ }^{7-8}$

Chemical reduction, electrochemistry, sonochemistry and laser ablation are versatile methods for synthesis of AgNPs. If produced in low concentration AgNPs colloidal dispersions are stable for months. However, the concentrated dispersions, usually applied to achieve the desired antibacterial effect, require stabilizers due to NP coalescence. ${ }^{9}$ In this light, stabilizing agents for particle surface passivation are explored to reduce the AgNPs aggregation. Although these agents vary from simple molecules to polymers, their common feature is the hydrophobic interaction with NPs surface. ${ }^{10-11}$ Some frequently reported AgNPs stabilizers are 
cetylammonium bromide, tetrabutylammonium acetate, poly(vinylpirrolidone) and biopolymers, such as chitosan. ${ }^{12}$

Due to their structural diversity and chemical functionality, biopolymers are considered advantageous for stabilization of metal NPs, in addition to being biocompatible and biodegradable. For example, those bearing amino moieties are intrinsically antimicrobial and impart positive charge onto the metal surface to lower the particles aggregation over a wide $\mathrm{pH}$ range, thereby extending the shelf life of these systems. ${ }^{13-14}$ Moreover, the inclusion of amino functionalities brings about reactivity to the otherwise inert metal NPs, necessary for permanent immobilization of the hybrid biopolymer-metal NPs onto different surfaces. Covalent immobilization on solid substrates is not only employed to facilitate the recovery, but also the NPs reuse. ${ }^{13,15}$

One potentially attractive and largely available material for AgNPs immobilization is the residual cork, mainly from cork stoppers industry in Mediterranean countries. Granulated or powdered cork particles have remarkable sorption capacity due to their porous surface and chemical composition, ${ }^{16-17}$ and thus can be used for adsorptive removal of organic pollutants and heavy metals in waste waters. The chemical composition of cork depends on its origin and varies between 40-60 \% suberin, 10-50\% lignin, 5-15\% polysaccharides and 10-25\% extractable components including waxes and tannins. ${ }^{18-19}$ Lignin, suberin and tannin molecules possess large number of phenolic moieties, which can be enzymatically oxidized into reactive quinones that can further react with nucleophiles such as the amino groups of the biopolymers via 1,4-Michael addition or Schiff base formation. The enzymatic coupling on cork of the hybrid biopolymer-NPs antibacterial systems offer advantages over chemical conjugation in terms of high specificity and mild reaction conditions. Phenoloxidases, such as tyrosinases and laccases, are enzymes capable 
to promote the grafting process. ${ }^{20}$ These enzymatic tools have been shared by other applications developed in our group, including lignin-based adhesives for floor coverings,${ }^{21}$ antimicrobial and shrink-resistant wool, ${ }^{22}$ permanent dyeing of cotton, ${ }^{23}$ antifouling coatings on urinary catheters, ${ }^{24}$ and biopolymer hydrogels for wound dressing ${ }^{25}$ thus demonstrating the versatility of the enzymatic grafting approach.

In this study AgNPs colloidal dispersions were synthesized by reduction of $\mathrm{AgNO}_{3}$ with $\mathrm{NaBH}_{4}$ and simultaneously doped with two amino-functional biopolymers: chitosan and 6-deoxy-6-( $\omega-$ aminoethyl) aminocellulose. In this reaction the chemical reagent can be omitted because both $\mathrm{CS}$ and $\mathrm{AC}$ have been used as combined reducing and capping agents. ${ }^{26-27} \mathrm{We}$ used $\mathrm{NaBH}_{4}$ as a fast reducing agent in order to avoid high temperatures in the reaction and/or the assistance of an additional technology required for the $\mathrm{Ag}^{+}$reduction. Our goal was to permanently graft the hybrid Ag-biopolymer systems on the surface of cork in which case the non-attached particles and the excess of $\mathrm{NaBH}_{4}$ are easily removed by thorough washing. Therefore, laccase-assisted oxidation of the phenolic structures in cork was used to covalently immobilize the hybrid biopolymer-AgNPs on the cork surface via reaction with the amino groups in biopolymers. The antimicrobial activity of the green-functionalized cork was evaluated against Escherichia coli and Staphylococcus aureus, in order to assess its potential as a filter to reduce these common pathogenic indicators of pollution in constructed wetlands. 


\section{Experimental section}

\subsection{Materials, reagents and bacteria}

Granulated cork with mean particle size of $\sim 0.5 \mathrm{~cm}$ was provided by the Catalan Cork Institute. Silver nitrate $\left(\mathrm{AgNO}_{3}\right)$, sodium borohidride $\left(\mathrm{NaBH}_{4}\right)$, hydrochloric acid, sodium hydroxide and ethanol were of analytical grade, purchased from Sigma-Aldrich (Spain). Laccase (EC 1.10.3.2 Trametes sp. laccase, Laccase L603P) was provided by Biocatalysts, UK. Laccase activity was determined by oxidation of $5 \mathrm{mM}$ 2,2'- azino-bis(3-ethylbenzthiazoline-6-sulfonic acid) (ABTS) substrate in $0.1 \mathrm{M}$ succinic acid/succinate buffer ( $\mathrm{pH} 5.0$ ), followed by an absorbance increase at $420 \mathrm{~nm}$ and $50{ }^{\circ} \mathrm{C}$, to obtain $0.14 \mathrm{U} \mathrm{mg}^{-1}$ protein, where one unit is defined as the amount of enzyme necessary to oxidize $1 \mu \mathrm{mol}$ of ABTS per $\min \left(\varepsilon_{420}=36,000 \mathrm{M}^{-1} \mathrm{~cm}^{-1}\right)$. Protein content was determined using the Bradford method, obtaining $0.125 \mathrm{mg}$ protein per $\mathrm{mg}$ solid. The two amino-containing biopolymers used in the study were medical grade chitosan $(\mathrm{CS}, \sim 15 \mathrm{kDa}$, DDA $87 \%$ ) obtained from Kitozyme (Belgium), and 6-deoxy-6-( $\omega$-aminoethyl) aminocellulose (AC, $\sim 15 \mathrm{kDa}$ ). Microcrystalline cellulose (Fluka, Avicel PH-101) dried at $105{ }^{\circ} \mathrm{C}$ for $2 \mathrm{~h}$ was used for the AC preparation. First, tosyl cellulose was prepared using the synthesis method of Rahn et al., ${ }^{28}$ obtaining the product with a degree of substitution (DS) of 0.8. Ethylendiamine was then added in excess and the mixture was stirred for $3 \mathrm{~h}$ at $100{ }^{\circ} \mathrm{C}$. After precipitation in $\mathrm{H}_{2} \mathrm{O}$ the conjugate was filtered, washed several times with isopropanol and $\mathrm{H}_{2} \mathrm{O}$, and finally dried in vacuum. Gram-positive Staphylococcus aureus (S. aureus, ATCC 25923) and Gramnegative Escherichia coli (E. coli, ATCC 25922) were used for the antimicrobial assays. Plate count agar and other reagents for cell culture studies were purchased from Sigma-Aldrich unless otherwise specified. 


\subsection{Methods}

\section{Preparation of biopolymer-doped Ag nanoparticles}

Biopolymers were dissolved to reach $0.8 \%(\mathrm{w} / \mathrm{v})$ in $1 \% \mathrm{CH}_{3} \mathrm{COOH}(\mathrm{CS})$ and distilled water (AC). The $\mathrm{pH}$ of the solutions were adjusted to 5 by adding $2 \mathrm{M} \mathrm{NaOH}$ in case of $\mathrm{CS}$ and $1 \mathrm{M}$ $\mathrm{HCl}$ in case of AC. AgNPs synthesis was carried out by chemical reduction of $\mathrm{Ag}^{+}$to elemental Ag using $\mathrm{NaBH}_{4}\left(17.5 \mathrm{mg} / \mathrm{mL}\right.$ in $\left.\mathrm{dH}_{2} \mathrm{O}\right)$, starting from aqueous solutions of $\mathrm{AgNO}_{3}(5 \mathrm{mg} / \mathrm{mL})$ in presence of $\mathrm{CS}$ or AC. First, $2 \mathrm{~mL}$ of $\mathrm{NaBH}_{4}$ was added to $30 \mathrm{~mL}$ of a biopolymer solution under vigorous stirring, and then $\mathrm{AgNO}_{3}$ was slowly added to reach the final volume of $50 \mathrm{~mL}$.

\section{Characterization of biopolymer-doped Ag nanoparticles}

The hybrid structures were characterized using $\zeta$ potential for the particles charge, dynamic light scattering (DLS) for the mean particle size, UV-Vis spectrophotometry to check for the presence and intensity of the AgNPs surface plasmon resonance (SPR) band, and scanning transmission electron microscopy (STEM) to visualize the particles and evaluate their stability in the obtained dispersions. $\zeta$ potential was measured using Malvern ${ }^{\circledR} Z$ Zetasizer Nano ZS, DLS measurements were performed using DL135 Particle Size Analyzer (Cordouan Technologies, France). Three samples of each AgNPs dispersion (in absence and presence of CS of AC) were processed acquiring 5 measurement cycles with $1 \%$ signal-to-noise ratio. The data were analyzed using NanoQ 1.2.1.1 software. UV-Visible spectra were recorded between 300-600 nm for the dispersions diluted 100 times in distilled water using a Cary 100 Bio spectrophotometer (Varian). The AgNPs in the dispersions were visualized by a Zeiss Neon FIB microscope (Carl Zeiss, 
Germany) in STEM mode operating at $30 \mathrm{kV}$ acceleration voltage. The samples for observation were drop-casted on a TEM holey carbon grid.

\section{Immobilization of the hybrid biopolymer-doped AgNPs onto cork}

Prior to the treatment with biopolymer-doped AgNPs, the cork granules were cleaned with aqueous solution of $\mathrm{HCl}\left(\mathrm{pH}\right.$ 2), distilled $\mathrm{H}_{2} \mathrm{O}, \mathrm{NaOH}$ solution (pH 10) and finally with $96 \%$ $\mathrm{EtOH}$, and dried at $60{ }^{\circ} \mathrm{C}$ for $12 \mathrm{~h}$. Thereafter, $1 \mathrm{~g}$ of the material was placed in a mixture containing $18 \mathrm{~mL}$ of AgNPs dispersion (pure, doped with CS or doped with AC - immediately after their preparation), $18 \mathrm{~mL}$ of $0.1 \mathrm{M}$ succinic acid/succinate buffer $(\mathrm{pH}$ 5), and $0.5 \mathrm{~mL}$ of laccase (final concentration $0.1 \mathrm{U} / \mathrm{mL}$ ). The reaction was allowed to proceed for $24 \mathrm{~h}$ at $50{ }^{\circ} \mathrm{C}$ and $30 \mathrm{rpm}$ in a laboratory dying machine Ahiba (Datacolor). Control samples were also prepared using the same treatment conditions without laccase (in presence of hybrid biopolymerAgNPs) and with laccase and CS or AC alone (without AgNPs). The treated cork was washed thoroughly with water to remove the loosely fixed particles and $\mathrm{NaBH}_{4}$ from the material, and finally dried at $50^{\circ} \mathrm{C}$ for $12 \mathrm{~h}$.

Fourier transform infrared spectroscopy (FTIR) was used to analyze the surface of the treated cork. IR spectra of the samples were collected in the range of 4000-600 $\mathrm{cm}^{-1}$ using Perkin-Elmer Spectrum 100 (USA) equipped with universal ATR sampling accessory, performing 64 scans for each spectrum. Scanning electron microscopy (SEM) was performed to examine the morphology of the AgNPs-biopolymer hybrids immobilized onto cork. The micrographs with magnification $\times 30 \mathrm{~K}$ were obtained using a Zeiss Neon FIB microscope (Carl Zeiss, Germany) operating in SEM mode. 


\section{Antimicrobial activity of AgNPs embedded cork matrices}

The antimicrobial performance of treated cork matrices was assessed against E. coli and $S$. aureus using the dynamic shake ASTM E 2149-01 test, as described by Petkova et al. ${ }^{29}$ The results were expressed as $\log _{10}$ of colony forming units (CFU) per millilitre of buffer solution in the flask. Besides the unwashed matrices (only rinsed with water after the treatment), the antibacterial performance of the untreated and treated cork was evaluated after immersing those in water for 1,2 and 5 days at room temperature while stirring (100 rpm) to mimic the environment in which the materials are intended to be used, i.e. water flow in constructed wetlands. Finally, the antimicrobial activity after 5 days washing is also expressed as a reduction percentage of the survived bacteria colonies, calculated as follows:

Reduction $(\%)=(\mathrm{A}-\mathrm{B}) / \mathrm{A} \times 100$

where A represents the number of colonies (CFU) survived from the dispersion that was not in contact with cork and B represents CFU for the dispersions in contact with the untreated and functionalized cork material. These values were also compared with the cork enzymatically treated with only biopolymers (solutions not containing AgNPs). 


\section{Results and discussion}

\subsection{Characterization of AgNPs and AgNPs-biopolymers dispersions}

AgNPs were synthesized by a chemical reduction of $\mathrm{AgNO}_{3}$ with $\mathrm{NaBH}_{4}$ in absence and presence of biopolymers. In order to confirm the interactions between CS and AC with AgNPs during their synthesis, the $\zeta$ potential of pure AgNPs and biopolymer-doped AgNPs was measured. AgNPs doped with biopolymers displayed positive $\zeta$ potential values due to the presence of amino groups in the macromolecules that are protonated at $\mathrm{pH} 5-$ used for the AgNPs preparation (Table 1). Given that pure AgNPs display a negative potential, these results suggest the interactions between the AgNPs and the biopolymers.

The pure AgNPs also displayed notably smaller hydrodynamic radius than their homologues doped with biopolymers (Table 1). When measured by DLS, the increase in the NPs diameter in presence of biopolymers represents an indirect demonstration that the AgNPs are coated with macromolecules in thick layers. ${ }^{13}$ This is the opposite to a thin layer doping of noble metal nanoparticles in which case the change in the hydrodynamic radius is negligible. ${ }^{10}$ Also, in our study it is probable that the similar $\mathrm{Mw}$ of the biopolymers used induced comparable size increase after the AgNPs doping.

Table 1. $\zeta$ potential and mean hydrodynamic radius of AgNPs synthesized in absence and presence of CS and AC.

\begin{tabular}{|c|c|c|}
\hline Sample & $\zeta$ potential $(\mathrm{mV})$ & Mean particle size $(\mathrm{nm})$ \\
\hline AgNPs & $-25.3 \pm 0.8$ & $32.3 \pm 1.7$ \\
\hline AgNPs-CS & $41.5 \pm 0.7$ & $239.6 \pm 13.5$ \\
\hline
\end{tabular}


The interactions between the AgNPs and biopolymers were further confirmed by STEM analysis. Without doping the AgNPs were abundant in the dispersion and most of the particles displayed the sizes $\leq 30 \mathrm{~nm}$ (Fig. 1A). In contrast, the average sizes of the AgNPs doped with CS or AC, which were shaped in rod-like structures, exceeded $100 \mathrm{~nm}$, thereby confirming the DLS findings (Fig. 1B and 1C). The doped particles were well dispersed in the biopolymer templates forming aggregated complexes, apparently as a result of their sticking upon drying of the sample. The pure AgNPs precipitated in a matter of hours leaving very few particles in the dispersion (Fig. 1D). This is expected since the synthesis of nanoparticles by strong chemical reduction often results in agglomeration and precipitation of the colloids. In contrast, the hybrid structures of the biopolymer-doped AgNPs were stable and resisted the aggregation in dispersion and precipitation (Fig. 1E and 1F). STEM images together with the macroscopic observation of the dispersions (inset images) provided a clear proof that both CS and AC stabilized the AgNPs dispersions through the passivation of the particles surface. 

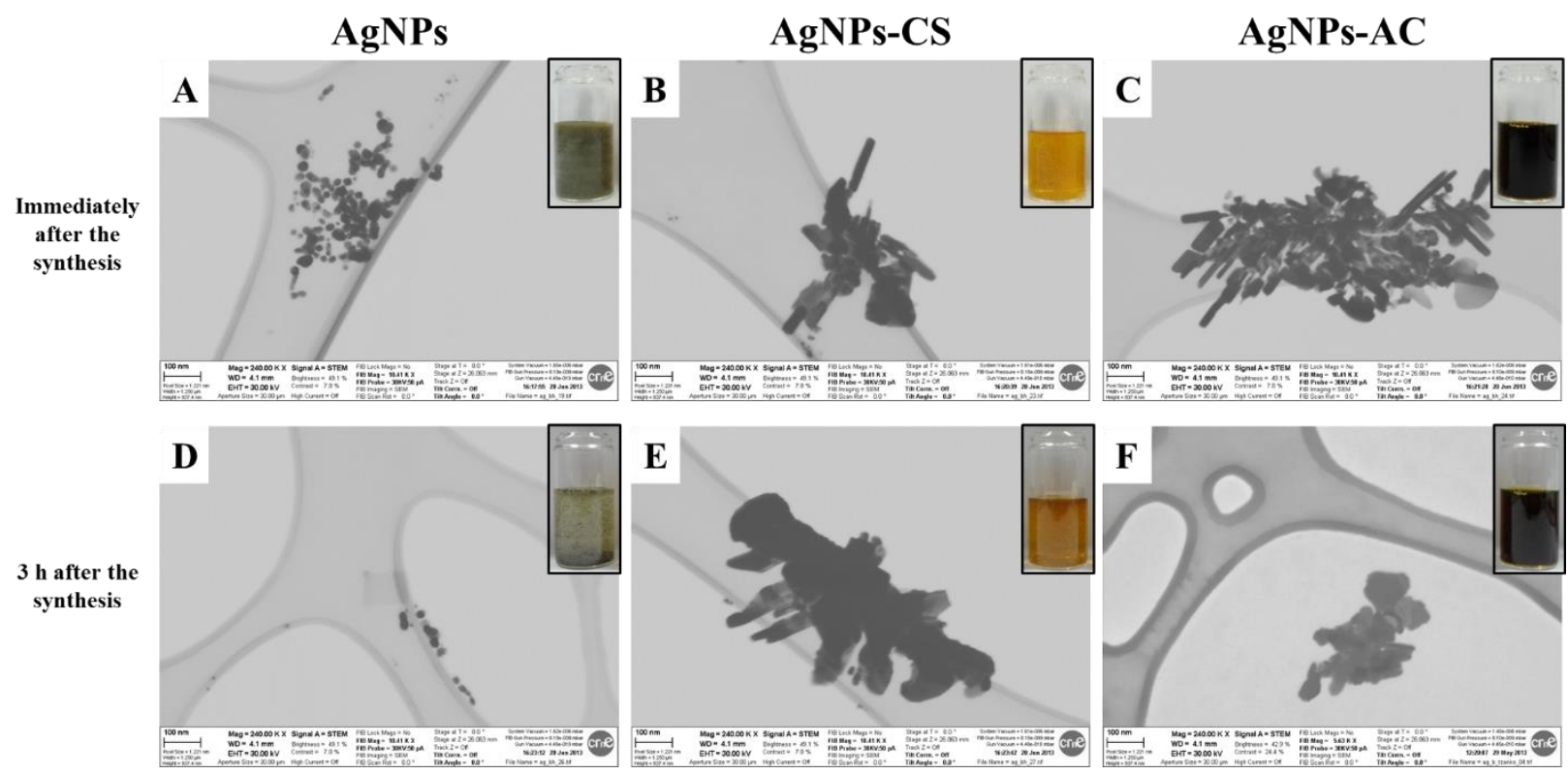

Fig. 1. STEM images of the AgNPs dispersions synthesized in absence and presence of CS and AC. The images were taken to evaluate the presence of nanoparticles in the dispersions immediately after the synthesis of the particles (images A, B and C) and after $3 \mathrm{~h}$ of their preparation (images D, E and F). The inset photographs represent the analyzed dispersions.

UV-Vis spectra were recorded for the pure AgNPs and the dispersions doped with CS and AC after $72 \mathrm{~h}$ of their synthesis. Since the dispersion prepared in absence of biopolymers was unstable and entirely precipitated during this time leaving the above solution clear, no characteristic for AgNPs absorption is observed in its spectrum in the 300-500 nm range (Fig. 2). In contrast, the SPR band was detected for the stable AgNPs dispersions prepared in presence of both CS and AC. The change in color of the dispersion and the consequent appearance of the bands between $400 \mathrm{~nm}$ (for AgNPs-AC) and 425 (for AgNPs-CS) implies the interaction between the biopolymers and AgNPs surface which modify their interaction with light. ${ }^{30}$ 
Besides the different wavelength position of the SPR peak, the intensity of AgNPs-AC was around 3 times higher than that of AgNPs-CS. The intensity of the band in the AgNPs systems where biopolymers are used as particle stabilizers varies as a function of the preparation conditions, as well as the amount of a biopolymer used for the particles doping. Nevertheless, despite the higher peak intensity of the AgNPs-AC system and the same starting concentrations of AC and CS, it still could not be concluded that this macromolecule interacts better with silver compared to $\mathrm{CS}$, especially in the case where the reduction of $\mathrm{Ag}^{+}$to elemental silver solely depends on the chemical compound $\left(\mathrm{NaBH}_{4}\right)$. Although the peak intensities are directly proportional to the amount of the AgNPs-biopolymer hybrids, these can only be compared between different samples of the same system (obtained varying the preparation conditions). For example, certain biopolymer concentrations can decrease the Ag particles surface exposure to light, which may be the reason for decrease or even the absence of the SPR band in such systems. ${ }^{31}$ If such scenario is applied to our case, we may even speculate on a better coverage of the AgNPs surface by CS compared to AC due to their strong interactions. Nevertheless, the strength of the organic-inorganic interactions as a function of the biomaterial structure goes beyond the scope of this manuscript.

Important from the technological point of view, the uniform color distribution throughout both AgNPs-CS and AgNPs-AC dispersions means that these hybrid systems were uniformly distributed in the dispersions, which broadens their application potential and facilitates exploitation of their functional properties. In addition, no precipitation was observed during several weeks after their synthesis, revealing sufficient stability of the systems for e.g. further surface functionalization of different materials. 


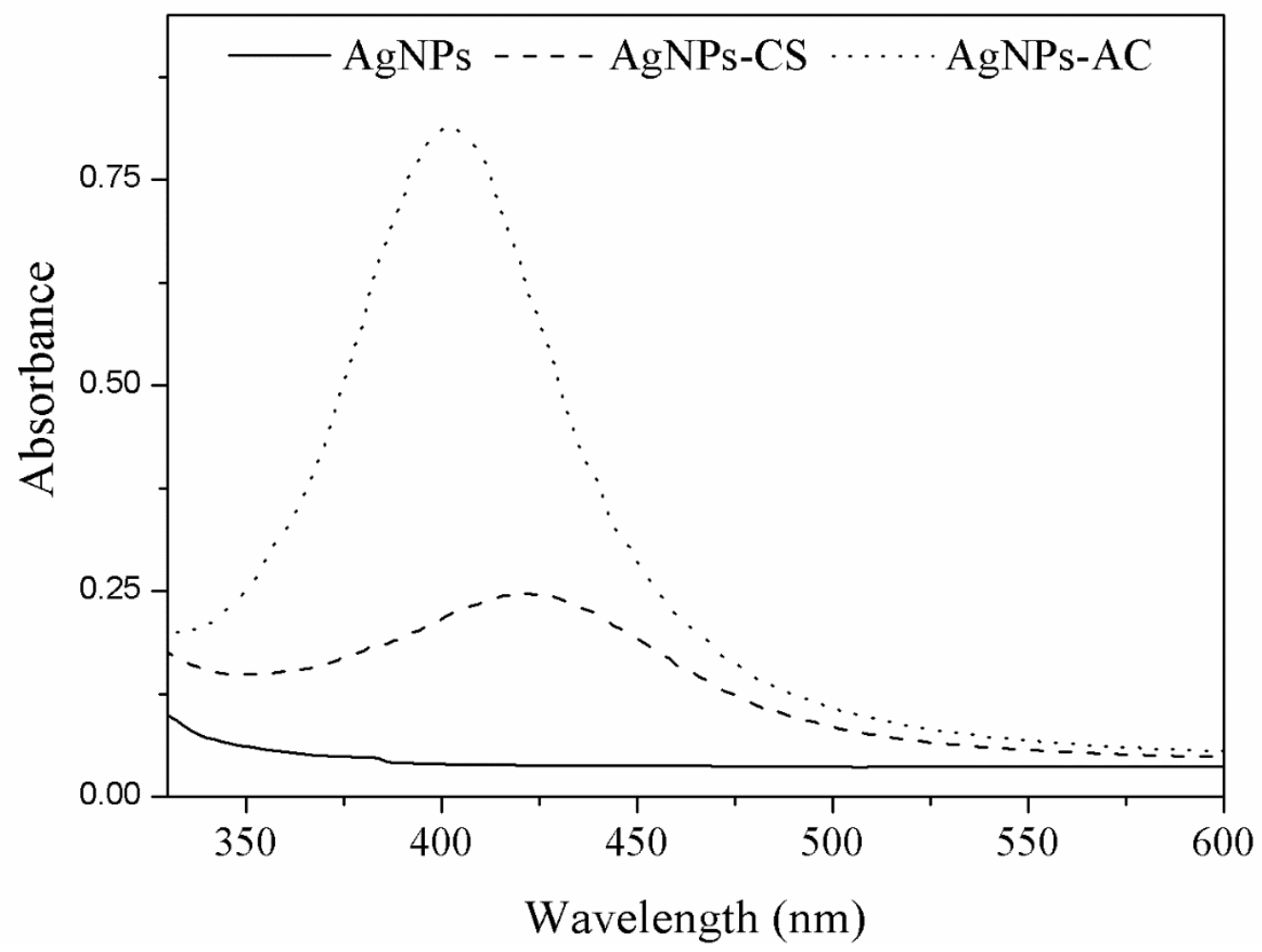

Fig. 2. UV-Vis spectra obtained for the pure AgNPs dispersion and the dispersions doped with CS and AC after $72 \mathrm{~h}$ of their synthesis.

\subsection{Enzymatic grafting of hybrid biopolymer-AgNPs on cork}

The characterization of the AgNPs and hybrid biopolymer-AgNPs using $\zeta$ potential, DLS, UVVis and STEM analysis, indicated that interactions between the particles and CS or AC occur. Doping of the AgNPs with these biopolymers provides the amino moieties on the particles surface necessary for the enzyme-catalyzed covalent immobilization of NPs onto cork. The used enzymatic immobilization approach is complex and may involve different interactions/reactions between the cork surface and used macromolecules, such as electrostatic interactions, hydrogen bonding and covalent linking between the laccase-activated cork moieties and CS or AC. In this work we targeted covalent bonding in order to permanently functionalize the cork surface with 
biopolymer-doped AgNPs using laccase-assisted immobilization which necessarily comprises: i) oxidation of the cork phenol groups (from suberin and lignin) to reactive quinones, and ii) reaction of the quinones with nucleophilic moieties (amino groups) in biopolymers through 1,4Michael addition or Schiff base formation, ${ }^{25}$ as illustrated on Scheme 1.

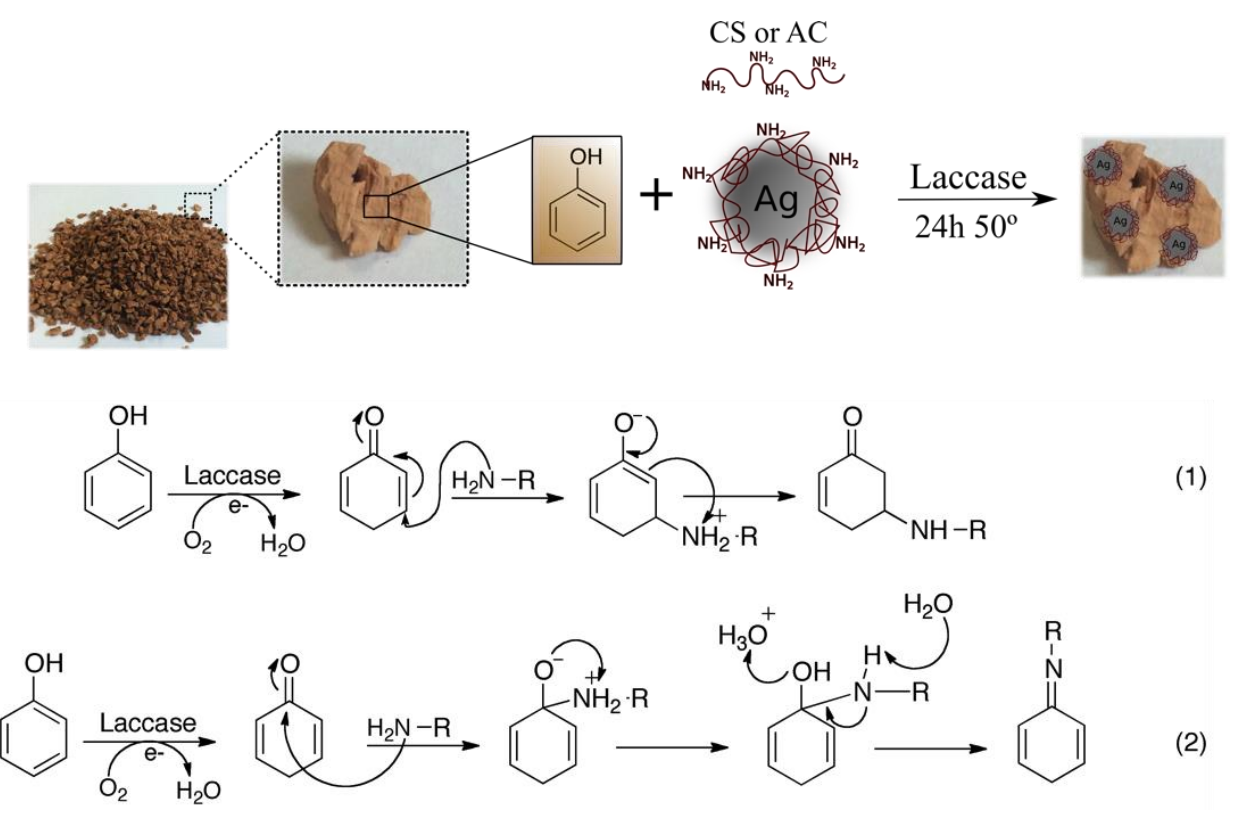

Scheme 1. Mechanism of immobilization of the hybrid AgNPs-biopolymers onto cork. Mechanisms above the scheme, show two possibilities of reaction: (1) Michael addition, (2) Schiff base formation.

FTIR spectra were recorded to verify whether the functionalization of the cork surface was successful. Prior to analysis, unmodified cork, cork enzymatically treated with pure AgNPs (immediately after their preparation) and cork treated with the hybrid biopolymer-AgNPs were subjected to thorough washing during which the samples were immersed in distilled water and vigorously stirred for 5 days. The water was changed each $24 \mathrm{~h}$ and the remaining solutions were checked for the presence of nanoparticles by optical microscopy using a Nikon Eclipse Ti microscope, with a 100x oil-immersion objective. After 4 days in water, no particles were 
detected in any of the remaining solution meaning that after this time there was no AgNPs leaching from the samples.

The unmodified cork and cork treated with pure AgNPs displayed characteristic for cork spectra with some of the most prominent bands as markers of: i) suberin at $1462 \mathrm{~cm}^{-1}, 1236 \mathrm{~cm}^{-1}$ and $1157 \mathrm{~cm}^{-1}$, its aliphatic chains at $2918 \mathrm{~cm}^{-1}$ and $2852 \mathrm{~cm}^{-1}$, and its ester groups at $1735 \mathrm{~cm}^{-1}$ and $721 \mathrm{~cm}^{-1}$, ii) lignin aromatics at $1600 \mathrm{~cm}^{-1}, 1510 \mathrm{~cm}^{-1}$ and $849 \mathrm{~cm}^{-1}$, and iii) cork polysaccharides at $1096 \mathrm{~cm}^{-1}$ and $1035 \mathrm{~cm}^{-1}$ (Fig. 3). ${ }^{32}$ Clear and comparable differences were found in the spectra of the cork treated with AgNPs-biopolymer hybrids. All peaks attributed to the cork suberin including the bands constituting the typical suberin fingerprint $\left(1462 \mathrm{~cm}^{-1}, 1236 \mathrm{~cm}^{-1}\right.$ and $\left.1157 \mathrm{~cm}^{-1}\right)^{33}$ decreased considerably after the enzymatic functionalization. Also the bands representing the lignin aromatics decreased or completely disappeared. Thus, both suberin and lignin from cork were involved in the functionalization reaction. At the same time, the differences in the spectra in polysaccharide regions between $1532-1681 \mathrm{~cm}^{-1}$ and $900-1120$ $\mathrm{cm}^{-1}$ suggest a successful biopolymer grafting, but this regions are rather complicated for deeper analysis. Instead, a shoulder peak was noticed at $1260 \mathrm{~cm}^{-1}$ in the spectra of the treated cork (especially pronounced for the cork treated with AgNPs-CS), which could be attributed to C-N stretching of aryl amides formed via Michael addition. ${ }^{25}$ Therefore, the FTIR spectra confirmed that the phenolic moieties from suberin and lignin in cork were covalently linked with CS or AC, where the reaction occurred predominantly via Michael addition. 


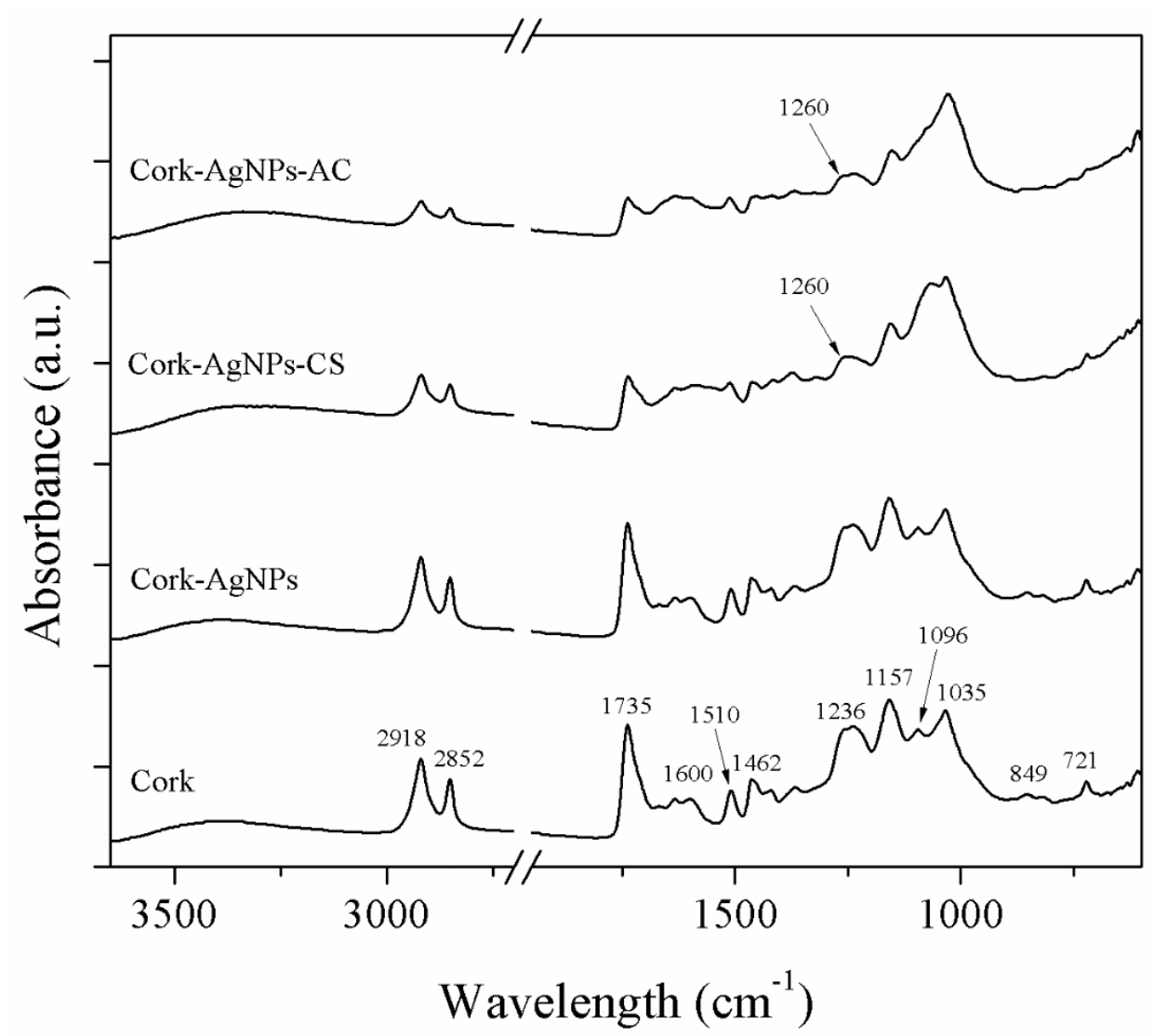

Fig. 3. FTIR-ATR spectra of untreated and enzymatically functionalized cork with AgNPs, AgNPs-CS and AgNPsAC dispersions.

SEM analysis was carried out in order to confirm the presence of AgNPs on the cork surface after the enzymatic treatment. On average, cork cells rarely exceed $50 \mu \mathrm{m}$ height, with a hexagonal face of $15-20 \mu \mathrm{m}$ and a thickness of 1-2 $\mu \mathrm{m} .{ }^{17}$ SEM images were thus taken inside the cells, first for the untreated cork without the NPs (Fig. 4A). The cell surface was not smooth and appeared granulated, where the remaining deposits, not removed during the cleaning procedure, were noticed. The same deposits were observed on the cork treated in presence of laccase with the AgNPs immediately after their synthesis (Fig. 4B). The AgNPs were thus not fixed on the cork surface or were removed during the immersion in water for 5 days. In contrast, particles and 
macromolecular structures were observed on the cork enzymatically embedded with the hybrid AgNPs-biopolymer systems even after extensive washing with water (Fig. 4C and 4D). This was especially the case of AgNPs-CS treated cork, which displayed a large amount of NPs on the surface. Both individual submicron particles and larger agglomerates could be visualized. A lower number of AgNPs was observed on the cork surface treated with AgNPs-AC system (marked with red arrows on Fig. 4D), but these were still fixed strong enough to resist the removal by thorough washing. A larger number of permanently deposited AgNPs was achieved via the laccase-assisted grafting of CS on cork.
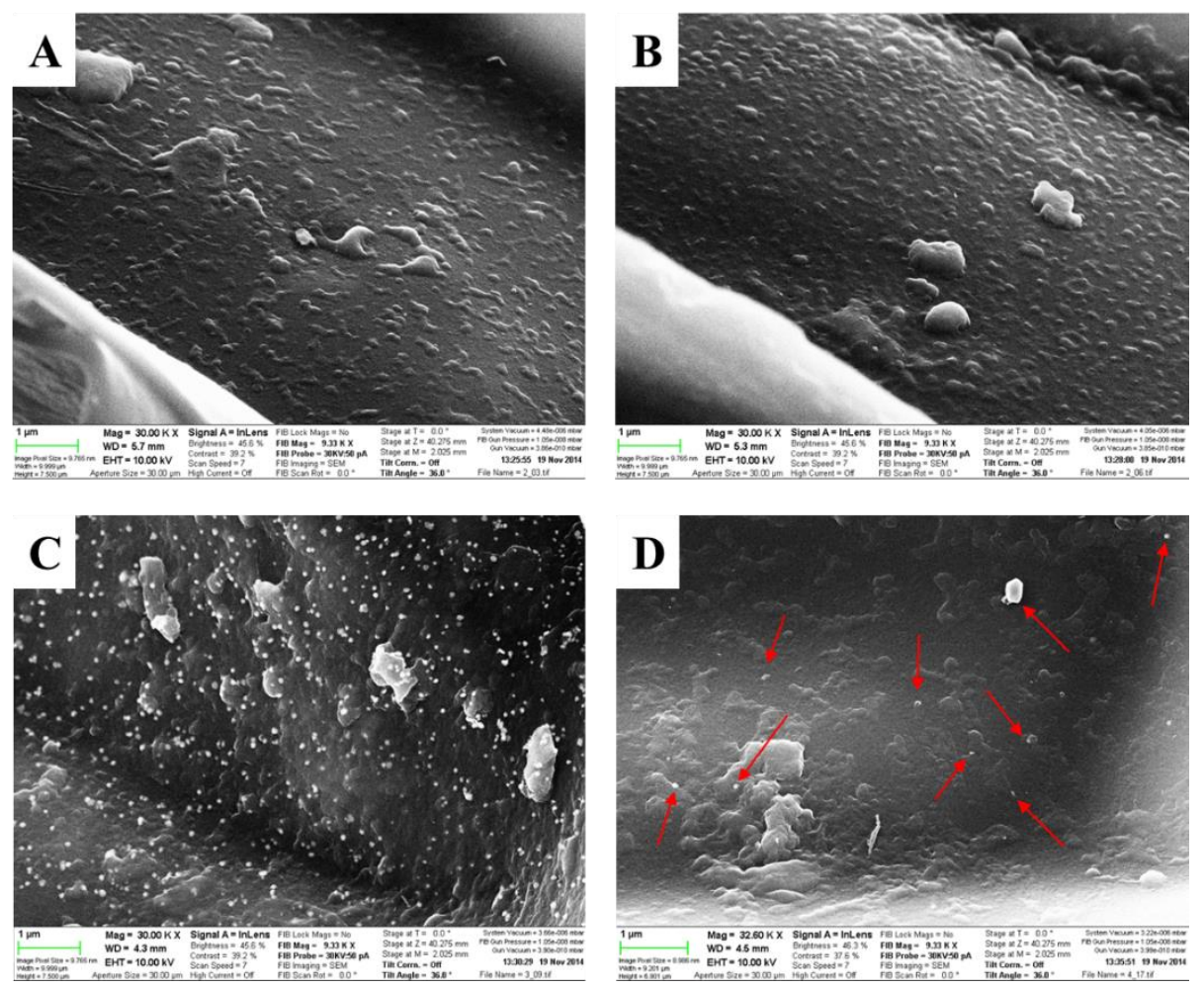

Fig. 4. SEM images of cork surface enzymatically treated in absence (A) and presence of nanoparticles: (B) pure AgNPs, (C) AgNPs-AC, and (D) AgNPs-CS. 


\subsection{Antimicrobial activity of AgNPs-embedded cork matrices}

The antibacterial activity of cork matrices functionalized with biopolymer-doped AgNPs was evaluated at different time points after immersing the materials in water up to 5 days. The results of the time-kill experiments showed that the cork matrices treated with pure Ag-NPs (immediately upon their preparation) fully inhibited the bacterial growth for both E. coli and $S$. aureus if no washing procedure was performed (Fig. 5). Although not expected in such extent, the antibacterial effect of cork-AgNPs sample could be explained by a moderate adsorption capacity of cork towards silver. ${ }^{34}$ In contrast, other materials such as nanoclay have been associated with AgNPs in absence of auxiliary molecules to impart diffusion-controlled antimicrobial activity, with long term impact. ${ }^{35}$ Adsorption of metals onto different sorbents may occur by physiosorption and chemisorption. ${ }^{17,36}$ Considering that the chemisorption involves the permanent modification through generation of new chemical bonds at the surface of a material and the progressive loss of the antibacterial effect of cork-AgNPs during 5 days in water, it is logical to conclude that this material maintained the activity whereas the physically adsorbed particles persisted on its surface. On the other hand, the cork matrices functionalized with AgNPs-CS and AgNPs-AC hybrids preserved most of the antibacterial effect (against $E$. coli) or fully retained the effect (against $S$. aureus) during the extensive washing procedure. Although it is probable that some of the hybrid structures were also physically adsorbed on surface (reflected in somewhat lower effect of the cork-AgNPs-CS and AgNPs-AC in case of E. coli after 5 days of washing), these results indirectly confirm the FTIR and SEM findings for the permanent functionalization of cork with AgNPs-biopolymer hybrids using the laccase-assisted approach. 

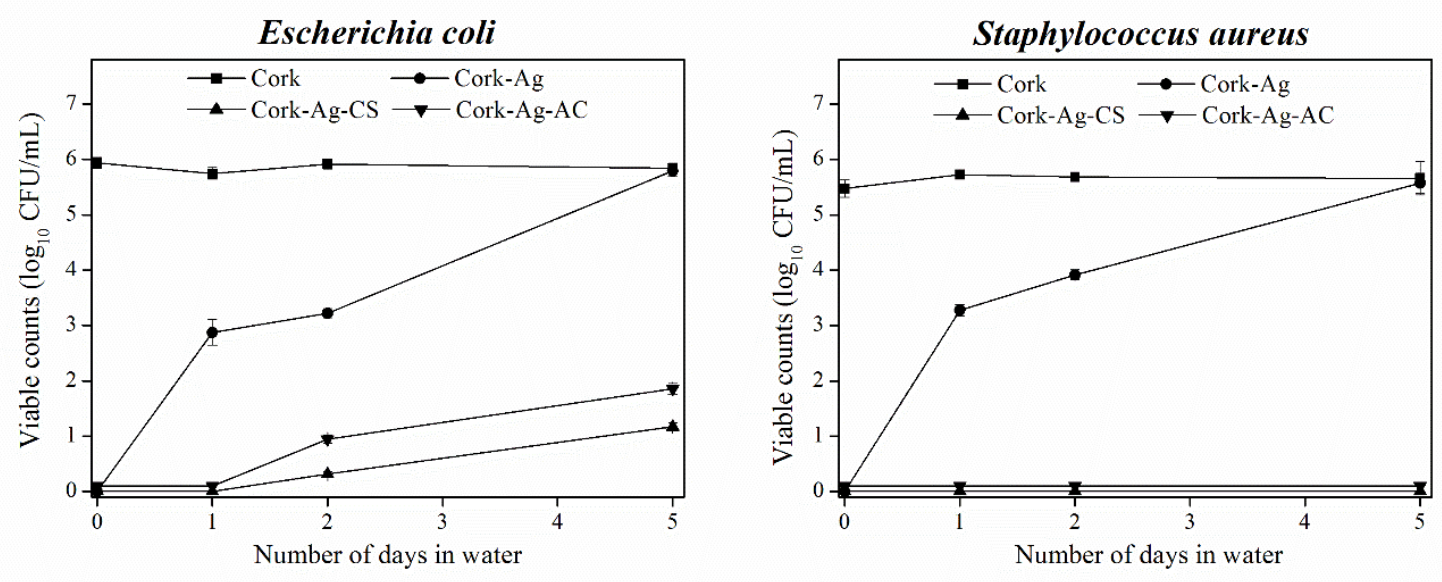

Fig. 5. Antimicrobial activity of untreated and enzymatically functionalized cork with AgNPs, AgNPs-CS and AgNPs-AC dispersions. The materials were subjected to the washing treatment in water (stirring $100 \mathrm{rpm}$ ) for up to 5 days, during which the antibacterial activity is evaluated at different time points.

The antimicrobial effect of cork-AgNPs-CS and cork-AgNPs-AC after 5-day washing was also compared to the matrices enzymatically functionalized with only CS and AC (without AgNPs), in order to reveal if the effect of the biopolymers was restricted only to the material functionalization. Calculated in percentage of bacterial inhibition compared to the effect of untreated cork, the matrices with enzymatically embedded hybrid AgNPs-CS and AgNPs-AC reduced respectively by $99.6 \%$ and $85.3 \%$ the growth of $E$. coli, whereas showing the full kill potential in the case of $S$. aureus (Table 2). Although the SEM images showed more AgNPs particles immobilized on cork in the case of AgNPs-CS, the antimicrobial efficiency of these two materials were comparable for the used bacteria inoculums. Such scenario did not allow to clearly distinguish the individual effects of AgNPs on one side and intrinsically antimicrobial CS or AC on another. Indeed, CS is a known while AC (a more economical substitute of CS) is an emerging antimicrobial macromolecule in different forms. ${ }^{37-38}$ Cork treated with CS and AC in 
absence of AgNPs did not show activity against E. coli, whereas the efficiency for $S$. aureus reduction was of $10.2 \%$ and $4.3 \%$, respectively. The antibacterial potential of these matrices is measured in the assay optimized to distinguish the effect of the cork embedded with AgNPs (small amount of the material and high bacteria count), which certainly possess higher antibacterial activity than the biopolymers. Thus, the absence or the low extent of the antibacterial potential of the matrices treated only with biopolymers is explained with the assay conditions applied. Moreover, a slightly higher antibacterial potential of the CS- treated compared to the matrices containing $\mathrm{AC}$ is attributed to better grafting of CS macromolecule compared to AC (concluded from the SEM images). Overall, the antibacterial efficiency of the cork grafted with hybrid AgNPs-biopolymers is largely dependent on the presence of silver. Nevertheless, some synergistic antibacterial effect of the AgNPs and the intrinsically antibacterial amino-functional biopolymers could still be envisaged, though the enhancement of antimicrobial activity in hybrid nanoscale architectures based on silver for long-term effect is not a new concept. ${ }^{39}$

Table 2. Antibacterial activity of enzymatically modified cork against Escherichia coli and Staphylococcus aureus after 5 days washing in water. The results are expressed in $\%$ of bacteria reduction compared to the untreated cork.

\begin{tabular}{ccc}
\hline Sample & E. coli reduction $(\%)$ & S. aureus reduction $(\%)$ \\
\hline Cork-AgNPs- CS & 99.6 & 100 \\
\hline Cork-AgNPs-AC & 85.3 & 100 \\
\hline Cork-CS & 0 & 10.2 \\
\hline Cork-AC & 0 & 4.3 \\
\hline
\end{tabular}




\section{Conclusions}

In this study, the laccase oxidation of the phenol moieties in cork into reactive quinones and their further reaction with nucleophilic amino groups in CS- or AC-doped AgNPs was exploited to impart durable antibacterial activity onto cork matrices. The role of CS and AC biopolymers in the hybrid NPs structures was three-fold in order to: (i) stabilize the AgNPs and prevent their aggregation, (ii) serve as an interface for permanent grafting of AgNPs on cork, and iii) synergistically improve the AgNPs antibacterial effect. The biocatalytically functionalized cork matrices with AgNPs-CS and AgNPs-AC efficiently reduced the Escherichia coli and Staphylococcus aureus growth during the course of 5 days. Such long-term stability and durability of the antimicrobial effect in conditions of continuous water flow, suggest the potential application of the functionalized cork matrices in constructed wetlands as an adsorbent for removal of wastewater impurities at the same time avoiding microbial contaminations. 


\section{References}

(1) Agudelo, R. M.; Peñuela, G.; Aguirre, N. J.; Morató, J.; Jaramillo, M. L. Simultaneous Removal of Chlorpyrifos and Dissolved Organic Carbon using Horizontal Sub-Surface Flow Pilot Wetlands. Ecol. Eng. 2010, 36, 1401-1408.

(2) Garcí, J.; Rousseau, D. P.; Morató, J.; Lesage, E.; Matamoros, V.; Bayona, J. M. Contaminant Removal Processes in Subsurface-Flow Constructed Wetlands: a Review. Crit. Rev. Environ. Sci. Technol. 2010, 40, 561-661.

(3) Li, Y.; Zhu, G.; Ng, W. J.; Tan, S. K. A Review on Removing Pharmaceutical Contaminants from Wastewater by Constructed Wetlands: Design, Performance and Mechanism. Sci. Total Environ. 2014, 468-469, 908-932.

(4) Casas-Zapata, J. C.; Ríos, K.; Florville-Alejandre, T. R.; Morató, J.; Peñuela, G. Influence of Chlorothalonil on the Removal of Organic Matter in Horizontal Subsurface Flow Constructed Wetlands. J. Environ. Sci. Health, Part B 2013, 48, 122-132.

(5) Phu, D. V.; Quoc, L. A.; Duy, N. N.; Hien, N. Q. Study of Incorporation of Silver Nanoparticles onto PE-g-PAAc Nonwoven Fabric by $\gamma$-Irradiation for Water Treatment. Radiat. Phys. Chem. 2013, 88, 90-94.

(6) Hossaina, F.; Perales-Perez, O. J.; Hwang, S.; Román, F. Antimicrobial Nanomaterials as Water Disinfectant: Applications, Limitations and Future Perspectives. Sci. Total Environ. 2014, 466-467, 1047-1059.

(7) Rai, M.; Yadav, A.; Gade, A. Silver Nanoparticles as a New Generation of Antimicrobials. Biotechnol. Adv. 2009, 27, 76-83.

(8) Sharma, V. K.; Yngard, R. A.; Lin, Y. Silver Nanoparticles: Green Synthesis and their Antimicrobial Activities. Adv. Colloid Interface Sci. 2009, 145, 83-96.

(9) Wang, W.; Gu, B. Preparation and Characterization of Silver Nanoparticles at High Concentrations. ACS Symp. Ser. 2009, 878, 1-14.

(10) Abdullin, T. I.; Bondar, O. V.; Shtyrlin, Y. G.; Kahraman, M.; Culha, M. Pluronic Block Copolymer-Mediated Interactions of Organic Compounds with Noble Metal Nanoparticles for SERS Analysis. Langmuir 2010, 26, 5153-5159.

(11) Bae, E.; Park, H.-J.; Park, J.; Yoon, J.; Kim, Y.; Choi, K.; Yi, J. Effect of Chemical Stabilizers in Silver Nanoparticle Suspensions on Nanotoxicity. Bull. Korean Chem. Soc. 2011, 32, 613-619.

(12) Reicha, F. M.; Sarhan, A.; Abdel-Hamid, M. I.; El-Sherbiny, I. M. Preparation of Silver Nanoparticles in the Presence of Chitosan by Electrochemical Method. Carbohydr. Polym. 2012, 89, 236-244.

(13) Ali, S. W.; Rajendran, S.; Joshi, M. Synthesis and Characterization of Chitosan and Silver Loaded Chitosan Nanoparticles for Bioactive Polyester. Carbohydr. Polym. 2011, 83, 438-446.

(14) Guo, Y.-B.; Wang, D.-G.; Liu, S.-H.; Zhang, S.-W. Fabrication and Tribological Properties of Polyelectrolyte Multilayers Containing in Situ Gold and Silver Nanoparticles. Colloids Surf., A 2013, 417, 1-9.

(15) Juan, L.; Zhimin, Z.; Anchun, M.; Lei, L.; Jingchao, Z. Deposition of Silver Nanoparticles on Titanium Surface for Antibacterial Effect. Int. J. Nanomed. 2010, 5, 261-267.

(16) Jové, P.; Olivella, M. À.; Cano, L. Study of the Variability in Chemical Composition of Bark Layers of Quercus Suber L. from Different Production Areas. BioResources 2011, 6, 18061814. 
(17) Pintor, A. M.; Ferreira, C. I.; Pereira, J. C.; Correia, P.; Silva, S. P.; Vilar, V. J.; Botelho, C. M.; Boaventura, R. A. Use of Cork Powder and Granules for the Adsorption of Pollutants: a Review. Water Res. 2012, 46, 3152-3166.

(18) Chubar, N.; Carvalho, J. R.; Correia, M. J. N. Cork Biomass as Biosorbent for Cu(II), Zn(II) and Ni(II). Colloids Surf., A 2003, 230, 57-65.

(19) Olivella, M. À.; Jové, P.; Bianchi, A.; Bazzicalupi, C.; Cano, L. An Integrated Approach to Understanding the Sorption Mechanism of Phenanthrene by Cork. Chemosphere 2013, 90, 1939-1944.

(20) Teixeira, L. S. M.; Feijen, J.; Blitterswijk, C. A. v.; Dijkstra, P. J.; Karperien, M. Enzyme-Catalyzed Crosslinkable Hydrogels: Emerging Strategies for Tissue Engineering. Biomaterials 2012, 33, 1281-1290.

(21) Aracri, E.; Blanco, C. D.; Tzanov, T. An Enzymatic Approach to Develop a Lignin-Based Adhesive for Wool Floor Coverings. Green Chem. 2014, 16, 2597-2603.

(22) Hossai, K. M. G.; González, M. D.; Juan, A. R.; Tzanov, T. Enzyme-Mediated Coupling of a Bi-Functional Phenolic Compound onto Wool to Enhance its Physical, Mechanical and Functional Properties. Enzyme Microb. Technol. 2010, 46, 326-330.

(23) Blanco, C. D.; González, M. D.; Monmany, J. M. D.; Tzanov, T. Dyeing Properties, Synthesis, Isolation and Characterization of an in Situ Generated Phenolic Pigment, Covalently Bound to Cotton. Enzyme Microb. Technol. 2009, 44, 380-385.

(24) Blanco, C. D.; Ortner, A.; Dimitrov, R.; Navarro, A.; Mendoza, E.; Tzanov, T. Building an Antifouling Zwitterionic Coating on Urinary Catheters Using an Enzymatically Triggered Bottom-Up Approach. ACS Appl. Mater. Interfaces 2014, 6, 11385-11393.

(25) Rocasalbas, G.; Francesko, A.; Touriño, S.; Fernández-Francos, X.; Guebitz, G. M.; Tzanov, T. Laccase-Assisted Formation of Bioactive Chitosan/Gelatin Hydrogel Stabilized with Plant Polyphenols. Carbohydr. Polym. 2013, 92, 989-996.

(26) Huang, H.; Yuan, Q.; Yang, X. Preparation and Characterization of Metal-Chitosan Nanocomposites. Colloids Surf., B 2004, 39, 31-37.

(27) Cheng, F.; Betts, J. W.; Kelly, S. M.; Schaller, J.; Heinze, T. Synthesis and Antibacterial Effects of Aqueous Colloidal Solutions of Silver Nanoparticles Using Aminocellulose as a Combined Reducing and Capping Reagent. Green Chem. 2013, 15, 989-998.

(28) Rahn, K.; Diamantoglou, M.; Klemm, D.; Berghmans, H.; Heinze, T. Homogeneous Synthesis of Cellulose p-Toluenesulfonates in N,N-Dimethylacetamide/LiCl Solvent System. Angew. Makromol. Chem. 1996, 238, 143-163.

(29) Petkova, P.; Francesko, A.; Fernandes, M. M.; Mendoza, E.; Perelshtein, I.; Gedanken, A.; Tzanov, T. Sonochemical Coating of Textiles with Hybrid ZnO/Chitosan Antimicrobial Nanoparticles. ACS Appl. Mater. Interfaces 2014, 6, 1164-1172.

(30) George, J.; Kumar, R.; Sajeevkumar, V. A.; Ramana, K. V.; Rajamanickam, R.; Abhishek, V.; Nadanasabapathy, S.; Siddaramaiah. Hybrid HPMC Nanocomposites containing Bacterial Cellulose Nanocrystals and Silver Nanoparticles. Carbohydr. Polym. 2014, 105, 285292.

(31) Tran, H. V.; Tran, L. D.; Ba, C. T.; Vu, H. D.; Nguyen, T. N.; Pham, D. G.; Nguyen, P. $\mathrm{X}$. Synthesis, Characterization, Antibacterial and Antiproliferative Activities of Monodisperse Chitosan-based Silver Nanoparticles. Colloids Surf., A 2010, 360, 32-40.

(32) Lopes, M. H.; Barros, A.; Neto, C. P.; Rutledge, D. N.; Delgadillo, I.; Gil, A. M. Variability of Cork from Portuguese Quercus Suber Studied by Solid-State 13C-NMR and FTIR Spectroscopies. Biopolymers 2001, 62, 268-277. 
(33) Lopes, M. H.; Neto, C. P.; Barros, A.; Rutledge, D. N.; Delgadillo, I.; Gil, A. M. Quantitation of Aliphatic Suberin in Quercus Suber L. Cork by FTIR Spectroscopy and SolidState 13C-NMR Spectroscopy. Biopolymers 2000, 57, 344-351.

(34) Hanzlík, J.; Jehlička, J.; Šebek, O.; Weishauptová, Z.; Machovič, V. Multi-Component Adsorption of $\mathrm{Ag}(\mathrm{I}), \mathrm{Cd}(\mathrm{II})$ and $\mathrm{Cu}(\mathrm{II})$ by Natural Carbonaceous Materials. Water Res. 2004, 38, 2178-2184.

(35) Girase, B.; Depan, D.; Shah, J. S.; Xu, W.; Misra, R. D. K. Silver-Clay Nanohybrid Structure for Effective and Diffusion-Controlled Antimicrobial Activity. Mater. Sci. Eng., C 2011, 31, 1759-1766.

(36) Witek-Krowiak, A.; Szafran, R. G.; Modelski, S. Biosorption of Heavy Metals from Aqueous Solutions onto Peanut Shell as a Low-Cost Biosorbent. Desalination 2011, 265, 126134.

(37) Genco, T.; Zemljič, L. F.; Bračič, M.; Stana-Kleinschek, K.; Heinze, T. Physicochemical Properties and Bioactivity of a Novel Class of Cellulosics: 6-Deoxy-6-Amino Cellulose Sulfate. Macromol. Chem. Phys. 2012, 213, 539-548.

(38) Fernandes, M. M.; Francesko, A.; Torrent-Burgués, J.; Carrión-Fité, F. J.; Heinze, T.; Tzanov, T. Sonochemically Processed Cationic Nanocapsules: Efficient Antimicrobials with Membrane Disturbing Capacity. Biomacromolecules 2014, 15, 1365-1374.

(39) Misra, R. D. K.; Girase B.; Depan, D.; Shah, J. S. Hybrid Nanoscale Architecture for Enhancement of Antimicrobial Activity: Immobilization of Silver Nanoparticles on ThiolFunctionalized Polymer Crystallized on Carbon Nanotubes. Adv. Eng. Mater. 2012, 14, B93B100. 


\section{Table of Contents}

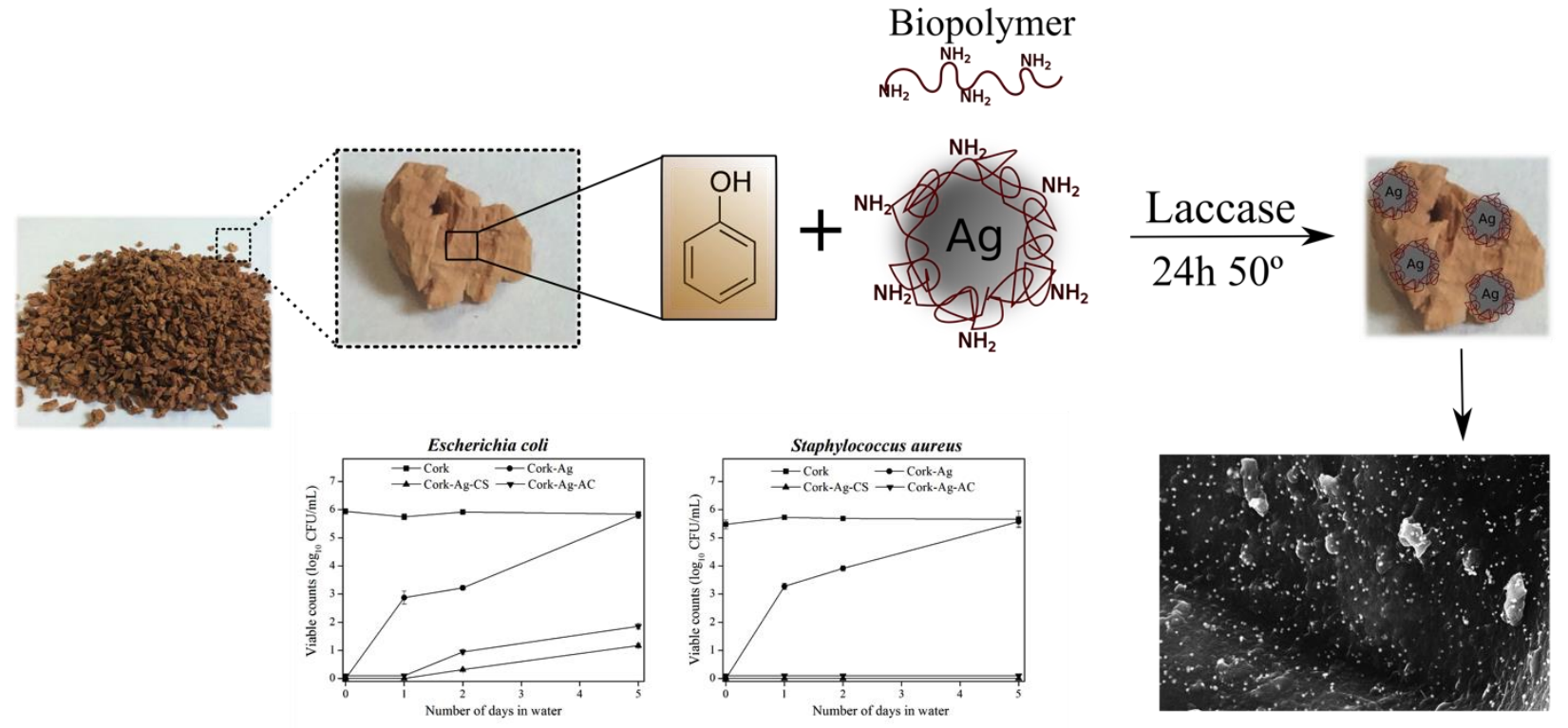

